\title{
Study on the Categorization Prototype Theory of Cognitive Linguistics
}

\author{
Zhao Huiyan ${ }^{1}$ \\ ${ }^{1}$ Anshan Normal University, Anshan, Liaoning P.R. China 114007 \\ a10013532@qq.com
}

Keywords: Prototype Theory, Categorization, Cognitive Linguistics

\begin{abstract}
Cognitive linguistics is a new discipline that has attracted the attention of various linguists in recent years. It is based on the cognitive method of psychology and brings the problems in linguistics into new explanation. In this paper, the typical theory of categorization of cognitive psychology is applied to linguistics, and some aspects of syntax, lexicology, pragmatics and semantics in linguistics are expounded.
\end{abstract}

\section{Introduction}

The term category is a widely used and ambiguous term in cognitive linguistics. A thing and its similar members (such as apples) can constitute a category, a class of things (such as movement) and its inclusion can constitute a category. We divide the categories of objects and things into one category because we feel that they are connected to some extent. The ability of this sorting enables us to be in contact with the surrounding environment without being confused by its complexity and diversity. "First, the categorization of objects can reduce the complexity of the surrounding environment. Scientists have developed the world has seven million kinds of distinguishable colors. If we think that all these colors are independent and unique, then we spend the time we have to learn the names of these colors. When we classify these distinguishable different colors, we only need to remember the names of their categories. Second, categorization is the world's objects are identified when we classify an object into a well-known category, such as a dog, a chair, a letter, etc., we usually think that we have known the object. Third, the establishment of the category reduces the complexity we can use the existing knowledge to understand new things. Fourth, categorization allows us to decide what can constitute a correct behavior. When we can sort out new things, they have to understand, we can use existing knowledge to understand new things. If you want to eat wild mushrooms, you must be able to distinguish between toxic and non - toxic wild mushrooms, and eating wild mushrooms is clearly not a correct act. Fifth, categorization allows us to arrange and contact the object class. Although the classification itself is an effective way of organizing knowledge, the category can also have further upper and lower off cuts. For example, the chair category, the high back the chair is the lower category, and the furniture is its upper category, which forms a ladder, the furniture contains a chair, and the chair contains a high back chair.

\section{The Characteristics of Cognitive Linguistics}

Determine the meaning of the category and its number is uncertain, relative to the cognitive needs of people and change. For example, when you compare snakes and dogs, you can think of "no legs" is one of the properties of the snake, but when you distinguish between snakes and maggots, "eyes, teeth and joints" has become a snake's property.

Features are also central, important distinction between attributes and marginal, non-important attributes of the points, the center attribute has a greater distinction, the edge of the property and the adjacent category attributes cross.

The members of the category have overlapping attributes, that is, all members enjoy some 
common attributes, the formation of family similarity. Where all members share the properties are with coherence. In other words, the existence of a property may indicate the coexistence of related attributes, such as "feathers" and "hard mouth, eggs, flying" is related.

The status of members is not equal with members of the center and the edge of the division with more common attributes of the members is members of the center. It should be noted that, while "prototypes" are often used to refer to the best members or typical representatives within the category, the more precise meaning of the prototype is the graphical representation of the core of the category, which is a categorical cognitive reference pointand its best members are only examples of the prototype. Prototype that the concept and the image of feeling linked, there is a certain truth. The cognitive category is stored in the brain as a cognitive concept, and its external manifestation is the word in the language. Therefore, we can think that the cognitive category and the meaning is the same; and the scope can be extended, such as the song can be used to refer to the "chairman", cup can be used to refer to the trophy, etc., to expand the scope of meaning, the formation of polysemy The expanded polysemy has been expressed as a cross between multiple categories. Chair also refers to the "chair" and "chairman" has been involved in two categories. Cognitive linguistics not only to explore a single category, but also to explain the same word that different areas between the projection relationships.

\section{Categorical classics of Cognitive Linguistics}

The division of the word class is the basis of grammar, but also confused linguistics nearly a hundred years of language problems. So far, linguists basically still follow the ancient Latin class division method. But in the division of words, there have been many differences. Crystia and Quirk have noticed that there are some blurred phenomena in the word class. The typical members of a certain part of speech have more classification features, and the non-typical members have fewer features. The ambiguity of the word class exists not only in the category of word class also exist between the word class. Cognitive linguistics also admit its ambiguity, but more attention to explore the origin and essence of this phenomenon, that the class of members of the typical degree and its semantic content and function, so the semantic function must be studied from the meaning of the word class.

Word class is a kind of archetype, which is based on the similarity between words and words in the distribution of the family gathered. Since the words of the same word class do not necessarily have a distinguishing feature of a group of other words, we can not classify all the words and define different words by using the trade-offs between several distinguishing features as criteria. Words belonging to the same word class are typical members and atypical members. The typical members are the prototype of a class of words, which is the reference standard when the atypical members are classified. Its typical members often have a set of distribution characteristics in the distribution can be distributed through the typical members of the word classification and to the definition of different parts of the word. However, the differences in the distribution of typical members of different parts of speech are obvious, and the differences in the distribution of atypical members of different parts of speech are blurred, which leads to the complex situation where the words can be classified but difficult to classify. According to the categorization theory of archetypes, it is a realistic and comprehensive approach to classify the word and classify it by using the classification of the advantages and disadvantages of the word distribution.

The family similarity of the category of words is a metaphorical argument. Meaning that the words belonging to the same word class have different degrees of similarity in grammatical nature, some of which have more similarity in the distribution, they become a typical member of the class, and some words with other words in the There are fewer similarities in the distribution, and they become atypical members of this category. In other words, the word class is a prototype category, is based on the word and the word between the grammatical natures of the similarity and summarized. Now we draw on the theory of cognitive archeology to discuss the classification of words, and finally give a phonetic system based on the prototype theory. The word class is a class that reflects the grammatical function of the word, so the word class can only be divided according to the 
grammatical distribution of the word. Since the distribution of words is very complicated, many of them are common to different categories of words; thus linguists usually choose only one or two distinct distribution features as classification criteria. The division of speech is now based on the classical categorization theory as a logical background. For example, it is common to think that all members of a part of a word must have a common or no distribution feature, and that the distribution of different values can be used to define different words. And the default word class in the same word class is equal and there are no typical and non-typical members of the other. This pursuit of simple, neat scientific ideals in the actual division of words often do not work, because the word class is not exactly the characteristics of category. To a large extent the word class is the prototype category.

\section{Categorization of the typical theory and semantic study}

"The formation of the form of language and the people's understanding of it is the result of the categorization of the world in which we live, so categorization is the object of semantic care first." "Class theory" has the most valuable research on semantics so many linguists regard "class typical theory" as a semantic theory. The scope of the meaning of the meaning of the word, especially the "Department of the word" meaning of the meaning of the range is a typical and atypical semantic categories. orsch has conducted an experiment, she let 200 subjects to determine the extent to which the object can be seen as a typical member of a category, she chose furniture, vehicles, birds and other ten categories to furniture for the For example, she asked the subjects to make a rating of 60 kinds of furniture (a total of seven) to judge. The results show that the chair in the 60 furniture in the first place, the phone is the end, scattered between the two have a table, bed, bookshelves and television. This experiment is an experiment done by a psychologist, but it has important linguistic significance.

At the beginning of the language, the language becomes a sign of a specific signal (ie, sensory, representation), a word that identifies a particular individual. For example, the word "chair" only signs the chair that the subject sees or has been sitting on. Later, the word begins to mark a group of similar objects, such as "chairs" can include bamboo chairs, rattan chairs, wooden chairs and other chairs. But this is only the combination of words and representations, but the general characteristics of the object of the general, and thus can not form a concept. With the development of cognitive ability, people began to use words to stabilize the main properties of a class of objects to be summarized, he can give up the color, size, material and so on the difference "chair" the word as a variety of similar External attributes, functional attributes of the same class of symbols, and even from the specific object to use the word, which produced a preliminary concept. The conceptual structure of the word consists of two parts, one is the prototyping of the family with the similarity of the extension of the members, part of the membership is a summary of all members. The combination of the two forms a prototype of meaning. The formation of the concept of thinking completes the first step from concrete to abstract, so that the language symbol has a meaning. The concept is the result of knowledge of things and its structure is based on the structure of the domain. The meaning of language is not the thing itself, but the people of their general understanding, exists in the interaction between people and the environment. With the development of cognition, the concept has been expanded. Because the brain's cognitive activity is based on the memory of the knowledge on the basis of the most economical way, so, with the understanding of new things, the brain is always looking for the existing concept of memory, according to the new understanding of things physics, function and other attributes will be known with the things that have some kind of connection, its classification. In this way, the concept of the original formation has been expanded to form a larger semantic category. We are still "chair" as an example. With the development of society and the emergence of new things, people's awareness of the scope is of the expansion.

\section{Conclusion}

Class typical theory on the scope of the division and the essence of a new perspective and 
methods will inevitably bring about changes in language research. Class theory on the field theory and semantic analysis of the epistemological basis of the proposed new interpretation method, which originated from the theory of cognitive psychology has become the cornerstone of cognitive linguistics. It is produced in the field of psychology, mature in the field of linguistics, but its theoretical significance is not limited to the understanding of the nature of language. "If the traditional semantic category concept gives people a way of recognizing absolute knowledge and making people accustomed to knowing things with polarized way of thinking, class theory may help people to correct this deviation."

\section{References}

[1] Wu Shixiong, Chen Weizhen. Family Similarity of Semantic Category [J]. Foreign Language Teaching and Research. 1996 (04)

[2] Zhang Yihua. On the necessary and necessary conditions and the interpretation of the prototype theory [J] .Dages Study. 2001 (04)

[3] Wu Shixiong, Chen Weizhen. China fuzzy linguistics: review and forward [J]. Foreign Language Teaching and Research. 2001 (01)

[4] Zhao Yanfang, Zhou Hong. Cognitive Mechanism of Semantic Category and Word Meaning Evolution [J]. Journal of Zhengzhou University of Technology, 2000 (04)

[5] Zou Zhiyong. Typical theory and language in the typical type [J]. Shandong Foreign Language Teaching. 1999 (04)

[6] Yang Zhong, Zhang Shaojie. Journal of Foreign Languages Teaching and Research. 1998 (02) 